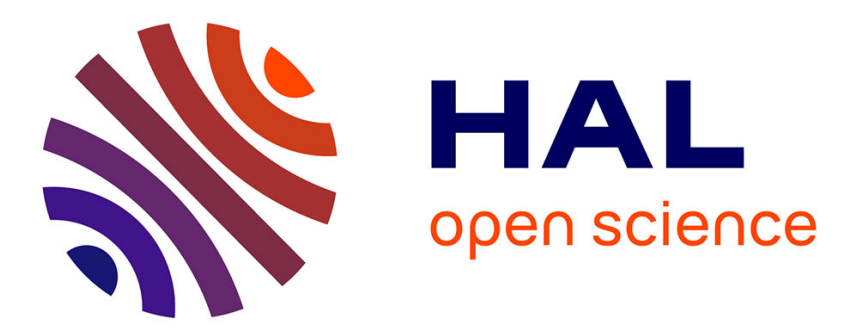

\title{
Doping-induced anisotropic lattice strain in homoepitaxial heavily boron-doped diamond
}

T. Wojewoda, Philipp Achatz, Luc Ortega, Franck Omnès, C. Marcenat, Emmanuel Bourgeois, Xavier Blase, François Jomard, Etienne Bustarret

\section{- To cite this version:}

T. Wojewoda, Philipp Achatz, Luc Ortega, Franck Omnès, C. Marcenat, et al.. Doping-induced anisotropic lattice strain in homoepitaxial heavily boron-doped diamond. Diamond and Related Materials, 2008, 17, pp.1302. 10.1016/j.diamond.2008.01.040 . hal-00761467

\section{HAL Id: hal-00761467 \\ https://hal.science/hal-00761467}

Submitted on 5 Dec 2012

HAL is a multi-disciplinary open access archive for the deposit and dissemination of scientific research documents, whether they are published or not. The documents may come from teaching and research institutions in France or abroad, or from public or private research centers.
L'archive ouverte pluridisciplinaire HAL, est destinée au dépôt et à la diffusion de documents scientifiques de niveau recherche, publiés ou non, émanant des établissements d'enseignement et de recherche français ou étrangers, des laboratoires publics ou privés. 


\title{
DOPING-INDUCED ANISOTROPIC LATTICE STRAIN IN HOMOEPITAXIAL HEAVILY BORON-DOPED DIAMOND
}

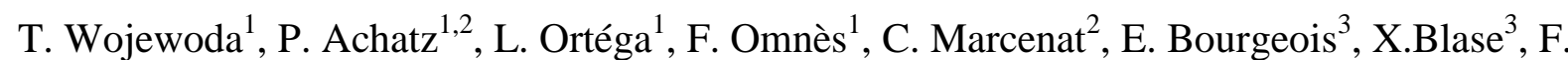 \\ Jomard $^{4}$, E. Bustarret ${ }^{1}$. \\ 1) CNRS, Institut Néel, BP166, 38042 Grenoble cedex 9, France \\ 2) CEA-Grenoble/DRFMC/SPSMS, 38054 Grenoble cedex 9, France \\ 3) LPMCN, CNRS and UCB, Domaine scientifique de la Doua, 69622 Villeurbanne, France \\ 4) GEMaC, CNRS and UVSQ, 1 place Aristide Briand, 92195 Meudon cedex, France
}

As a result of the larger covalent radius of boron $\left(\mathrm{r}_{\mathrm{B}}=0.88 \AA\right)$ when compared to that of carbon $\left(\mathrm{r}_{\mathrm{C}}=0.77 \AA\right)$, the introduction of substitutional boron into diamond leads to an expansion $\delta a / a$ of the lattice parameter. This has been found previously to follow a linear interpolation (Vegard law) as long as the boron content is lower than about 0.5 at.\% in MPCVD epilayers or 1.5 at.\% in HPHT bulk crystals.

Above those concentrations, the expansion is less pronounced than predicted by Vegard. In order to explain this effect, we have performed ab initio calculations on $\mathrm{C}: \mathrm{B}$ substitutional alloys. The results show that the presence of interstitial boron and of boron clusters is not necessary to explain the experimental data available in the literature. Moreover, quantitative estimates are proposed for the deformation potential of the valence band maximum and for the steric effect associated to boron pairing. We then apply these conclusions to discuss the different variations of $\delta a / a$ vs boron contents observed by high resolution XRD experiments performed on "insulating" and metallic (and superconducting) $\mathrm{p}^{++}$diamond epilayers grown by MPCVD on (100)- and (111)-oriented type Ib substrates, for which boron concentration profiles have been determined by Secondary Ion Mass Spectroscopy

Introduction

Boron is one of the few elements which may be readily introduced in substitution to carbon in the diamond lattice. Recently, both High Pressure High Temperature (HPHT) and Microwave Plasma-assisted Chemical Vapour Deposition (MPCVD) methods have led not only to borondoped polycrystals or epilayers but to boron-carbon substitutional alloys with a boron concentration on the order of 0.5 to $5 \%$. These materials are metallic and superconducting [16]. Their spectacular transport properties have led an increasing number of research groups to prepare and/or study such systems. Among such endeavours, a recent structural study has been devoted to the relationship between the lattice parameter a and the boron atomic density $\mathrm{n}_{\mathrm{B}}$ in HPHT single crystals [7] : as a result of the larger covalent radius of boron $\left(\mathrm{r}_{\mathrm{B}}=\right.$ $0.088 \mathrm{~nm}$ ) when compared to that of carbon $\left(\mathrm{r}_{\mathrm{C}}=0.077 \mathrm{~nm}\right)$, the introduction of substitutional boron into diamond leads to an expansion of the lattice parameter. This followed a linear interpolation (Vegard law [8]) as long as the boron content was lower than about 1.5 at.\% in these HPHT bulk crystals.

Above those concentrations, the expansion $\delta \mathrm{a} / \mathrm{a}$ has been found to be less pronounced than predicted by Vegard. This resolved a standing disagreement [6] between earlier reports [1,9] on epilayers grown by MPCVD, and paved the way to an estimate of the average boron concentration through double crystal XRD experiments [6]. Among others, the weaker expansion may be attributed to the contribution of free holes and to the negative deformation potentials at the valence band maximum, or to the occurrence of boron pairs or clusters. 
In order to distinguish between the effects which led to the observed deviations from Vegardtype behaviour, we performed ab initio structure calculations of $\mathrm{C}: \mathrm{B}$ substitutional model alloys as well as high resolution XRD experiments on insulating and metallic (and superconducting) $\mathrm{p}^{++}$diamond epilayers grown by MPCVD on (100)- and (111)-oriented type $\mathrm{Ib}$ substrates. In order to minimize the uncertainty on the absolute determination of boron atomic densities which has been a source of error in the past, the solid-state boron concentration profiles has been determined by carefully calibrated Secondary Ion Mass Spectrometry (SIMS).

We want to point out that despite their limitations in terms of number of atoms, or cell size, that can be studied, ab initio calculations provide valuable information on the structural and electronic properties of systems of interest. In particular, B-doped diamond structures with a well-defined doping level can be explored, at least in the large doping limit, that is in the few percent range. Further, the influence of the relative position of $\mathrm{B}$ atoms, either isolated or clustered as dimers, can be simulated in a realistic fashion. While the electronic and superconducting properties of B:C systems have been studied quite extensively, and by several groups, using such theoretical approaches [10-13], we shall focus here rather on the relationship between the doping rate and the lattice volume relaxation.

\section{Computational and experimental methods}

Our calculations are performed within a supercell planewave pseudopotential approach [14] to the density functional theory (DFT) within the local density approximation (LDA). In the supercell approach, boron atoms in substitution of carbon are repeated periodically throughout the crystal. This is the main drawback of the supercell technique, even though the effect of disorder can be somehow estimated by changing the unit cell shape and/or placing several atoms per cell at various relative positions, as described below. Beside the studies of electronic properties, such an approach has been used extensively to explore the elastic and plastic properties of pristine and doped $\mathrm{sp}^{3}$ column IV semiconductors such as diamond [10,15,16], silicon [17] and clathrates [17]. We adopt here a 50 Ry cutoff for the wavefunctions. As in Ref. [13], where the dimerisation of boron in diamond was sudied, two different supercells are used, a first one with 54 atoms which is composed of a $3 \times 3 \times 3$ repetition of the 2-atoms FCC diamond irreducible cell, and a 64-atoms cubic one obtained from a $2 \times 2 \times 2$ repetition of the conventional 8 -atoms cubic cell. For each cell, one to three substitutional $\mathrm{B}$ atoms are introduced in order to mimic various doping rates beyond the 1/64 at.\% doping ratio. Both atomic positions and lattice parameters are relaxed in order to minimize the total energy for each geometry. We study here only the "isotropic" relaxation by cancelling out all the diagonal components of the stress tensor.

The diamond samples were grown from $\mathrm{H}_{2} / \mathrm{CH}_{4} / \mathrm{B}_{2} \mathrm{H}_{6}$ gas mixtures by MPCVD at $2.45 \mathrm{GHz}$ onto (100)- or (111)-oriented diamond substrates held respectively at 830 and $900^{\circ} \mathrm{C}$. In the case of (100)-oriented substrates with a typical miscut angle of $0.3^{\circ}$, the total pressure was 30 hPa and the $\mathrm{CH}_{4}$ to $\mathrm{H}_{2}$ flow ratio was $4 \%$, the growth rate was on the order of $1 \mu \mathrm{m} / \mathrm{hr}$, and the boron solid-state incorporation was controlled by the $\mathrm{B} / \mathrm{C}$ atomic ratio in the gas phase, which was varied between 100 and $3000 \mathrm{ppm}$. A buffer layer of a few tens to a few hundreds of $\mathrm{nm}$ of non intentionally doped material was usually grown onto respectively (111)- and (100)-oriented substrates before $\mathrm{C}: \mathrm{B}$ homoepitaxial growth started. In the case of (111)oriented surfaces which had a typical miscut of $3^{\circ}$, pressure was held at $50 \mathrm{hPa}$, the growth rates were lower [13,19], and boron incorporation could be controlled either by the B/C gas ratio (from 100 to $6000 \mathrm{ppm}$ ) or by the $\mathrm{CH}_{4}$ to $\mathrm{H}_{2}$ flow ratio (from 0.1 to $0.6 \%$ ). In both cases, the incorporation efficiency of boron increased as the gas phase purity was improved in the silica-tube NIRIM-type deposition chamber (the main competitor for solid-state 
incorporation being $\mathrm{N}$ ). Details of the relationship between preparation conditions of the present epilayers, their boron content and their XRD patterns will be published elsewhere.

Absolute solid-state boron concentrations and film thicknesses were deduced from secondary ion mass spectroscopy (SIMS) analysis in a CAMECA IMS4f instrument by comparison with the yield of a diamond reference implanted with $210^{15} \mathrm{~B} / \mathrm{cm}^{2}$ which was measured in the same run with the same $10 \mathrm{keV}$ incident beam of $\mathrm{Cs}^{+}$ions, the extraction bias being $4.5 \mathrm{kV}$.

The X-ray Diffraction (XRD) data was collected around symmetrical Bragg reflexions ( $\{004\}$ or $\{111\}$ according to the orientation of the substrate) of both the epilayers and the substrates in a triple axis diffractometer using as a source the $\mathrm{Cu} \mathrm{K} \mathrm{K}_{\alpha 1}$ line selected through a double $\mathrm{Ge}$ crystal channel cut monochromator. In the case of reciprocal space mapping, another analyzer was located in front of the detector to increase selectivity.

\section{Calculated results and discussion of published experimental data}

The results of our ab initio calculations are compiled in Fig. 1, together with the experimental data points available in the literature [1,7]. Clearly, and in good agreement with the experimental results, in the "high" doping range where it applies, the theoretical analysis shows significant deviations from the simplest expression of Vegard law : $\delta a / a=\beta_{\text {size }} n_{B}$, with $\beta_{\text {size }}=\left(r_{B}-r_{C}\right) /\left(r_{C} n_{C}\right)$ where $n_{C}$ is the atomic density of diamond, i.e. $1.7610^{23} / \mathrm{cm}^{3}$, leading to a numerical value of $\beta_{\text {size }}=8.1210^{-25} \mathrm{~cm}^{3}$. Actually, the data points calculated for supercells containing from one to three isolated boron atoms are aligned rather close to a second linear interpolation also discussed by the authors of Ref. [7], where the average relaxed lattice parameter of the $\mathrm{C}: \mathrm{B}$ alloy is deduced from the weighted average of the specific atomic volumes of $\mathrm{C}\left(5.67 \mathrm{~A}^{3} /\right.$ atom $)$ and $\mathrm{B}\left(7.28 \AA^{3} /\right.$ atom $)$, yielding again to first order a linear variation of the type $\delta \mathrm{a} / \mathrm{a}=\beta_{\mathrm{vol}} \mathrm{n}_{\mathrm{B}}$. The slope $\beta_{\mathrm{vol}}=5.3810^{-25} \mathrm{~cm}^{3}$ is shown in fig. 1 to be in reasonable agreement with some of the experimental points. This coincidence led these authors to attribute the weakening of the lattice expansion to interstitial and/or aggregated incorporation of boron [7]. Because ab initio calculations yield also similar values for substitutional isolated boron atoms, we were led to question this assignment, and undertook to calculate the lattice expansion for supercells were boron atoms were in a nearest-neighbour geometry. Such boron pairs or $\mathrm{B}_{2}$ dimers, which do not contribute free holes to the crystal as isolated boron atoms do, have been found to be thermodynamically stable in p-type diamond, and their occurrence more probable at higher boron concentration, whereas $B_{n}$ clusters of more atoms and interstitial incorporation remained energetically unfavourable [13]. Again, the expansion calculated for such boron pairs is lower than predicted by Vegard, but it is stronger than that calculated for isolated boron atoms, and its variation with the density of $\mathrm{B}_{2}$ pairs $n_{\mathrm{BB}}$ is again found to be compatible (see fig. 1 ) with a linear expression of the type $\delta \mathrm{a} / \mathrm{a}=$ $\beta_{\text {pair }} \mathrm{n}_{\mathrm{BB}}$, with $\beta_{\text {pair }}=12.410^{-25} \mathrm{~cm}^{3}$. Please note that this value cannot explain the experimental results at "high" boron concentrations. In order to explain the low expansion values calculated for isolated substitutional boron atoms, another contribution has to be introduced, which describes the effect on the lattice parameter of the concentration $\mathrm{h}^{+}$of free carriers depleting the bonding states near the valence band maximum. This is often modelled by a (here negative) hydrostatic deformation potential $D_{p}$ at the top of the valence band [20], i.e. by a linear term which may be expressed as : $\beta_{\mathrm{p}} \mathrm{h}^{+}=\mathrm{D}_{\mathrm{p}} \mathrm{h}^{+} / 3 \mathrm{~K}, \mathrm{~K}$ being the bulk elastic modulus of diamond $\left(\mathrm{K}=4.4210^{7} \mathrm{Ncm}^{-2}\right)$. When all these terms are taken into account, Vegard law becomes :

$$
\delta \mathrm{a} / \mathrm{a}=\beta_{\text {size }} \mathrm{n}_{\mathrm{B}}+\beta_{\text {pair }} \mathrm{n}_{\mathrm{BB}}+\beta_{\mathrm{p}} \mathrm{h}^{+}
$$


Now, if we consider that to first order the slope $\beta_{\text {vol }}$ of the lower straight line drawn in fig. 1 is an adequate interpolation of the results of the calculations for systems free of boron pairs and where $n_{B}=h^{+}$, we can estimate $\beta_{p}=\beta_{\text {vol }}-\beta_{\text {size }}=-2.7410^{-25} \mathrm{~cm}^{3}$ and then deduce that $D_{p}=-2.26$ $\mathrm{eV}$, in good agreement with former band structure calculations [21] yielding $\mathrm{D}_{\mathrm{p}}=-2.4 \mathrm{eV}$.

\section{Experimental results and discussion}

A typical data set obtained at room-temperature on both a (100)- oriented and a (111)-oriented homoepitaxial film is represented by intensity contours in figs. 2 and 3 and shows that the down-shifted $\{400\}$ (resp. $\{111\}$ ) symmetrical diffraction peak originating from the epilayer has a lineshape very similar to that of the type Ib diamond substrate. In particular, the width of the rocking curve along the vertical axis (omega-scan) shows that the mosaicity of the HPHT substrate is maintained in the epilayer for both orientations, whereas the strain distribution represented by the lineshape along the horizontal axis ( 2 theta - omega scan) became somewhat broader in the epilayer. For each sample, the average perpendicular strain value $\delta \mathrm{a}^{\perp} / \mathrm{a}_{\mathrm{epi}} \perp \approx \delta \mathrm{a}^{\perp} / \mathrm{a}_{\mathrm{sub}} \perp$ is then deduced from the interplane distance, i.e. from the angular difference $\Delta \theta=\theta_{\text {epi }}-\theta_{\text {sub }}$ between the two Bragg peaks through the usual formula :

$$
\delta \mathrm{a} \perp / \mathrm{a} \perp=\Delta \theta / \tan \theta_{\text {sub }}
$$

Assuming the epitaxy to be coherent, pseudomorphic growth of $\mathrm{C}: \mathrm{B}$ on diamond substrates ensured that the lattice parameter in the plane of the epitaxial interface was conserved, so that, if the thickness of the layer was small compared to that of the substrate, all the strain induced by doping was observed along the stress-free growth direction probed by the present XRD experiments. Now, diamond being an elastically anisotropic material, the relationship between this perpendicular strain and the strain da/a of the epilayer in the "relaxed" state [9] depends on the crystallographic orientation of the layer :

$$
\delta \mathrm{a} / \mathrm{a}=\gamma_{\mathrm{hkl}} \delta \mathrm{a}_{\mathrm{epi}} \perp / \mathrm{a} \perp
$$

This strain in the relaxed state is the quantity that should be used for comparison to calculated values and other published data. It has been evaluated for two sets of epilayers grown as described above on top of (100)-oriented $(\gamma=0.8113)$ and on (111)-oriented $(\gamma=0.9136)$ type Ib substrates, and the results are shown in figs 4 and 5 . In each case, the difference between the two sets was that one set (full symbols) was grown under improved growth conditions with respect to the other (empty symbols) from the point of view of hydrogen purity. As seen in fig.4, in the case of (100)-oriented growth, the data points lie again between the two linear interpolations already mentioned, with a high proportion of samples following Vegard law up to about 1 at.\%, i.e. at higher concentrations than previously reported [1]. The trends at higher boron concentrations remain to be confirmed in other (100)oriented MPCVD grown epitaxial alloys. In the case of (111)-oriented epitaxy (see fig.5), the concentration range is about three times larger, and the experimental expansion values are always significantly lower than predicted by Vegard, even at relatively low boron content. In fact, many points lie close to the values predicted for full boron pairing. Although this is most likely a coincidence, it might indicate that under our (111)-oriented growth conditions such boron pairing is favoured as suggested previously by other authors [13,22]. Such pairs being electrically inactive, their presence would also explain the higher boron threshold density needed to obtain metallicity and superconductivity in our (111)-oriented epilayers when compared to (100)-oriented single crystal films [23]. 
Concluding remarks

We have confirmed that above $1.5 \pm 0.5$ at. $\%$, the presence of boron in diamond induced a lattice expansion significantly lower than that predicted by Vegard. Ab initio supercell calculations showed that the results could be explained taking into account the depletion of the bonding states at the top of the valence band associated to the presence of free carriers in such a metallic material. Confirming their relevance in the field of doped diamond, ab initio simulations further showed that boron pairing, although not contributing to doping, also induced a lattice expansion intermediate between that expected from Vegard law and that associated to free carriers. This last result may explain the XRD results obtained here on some $\{111\}$-oriented C:B epilayers.

\section{Acknowledgements}

Partial financial support by the Agence Nationale pour la Recherche through contract ANR05-BLAN-0282 is gratefully acknowledged.

\section{References}

[1] E. Bustarret, E. Gheeraert, K. Watanabe, phys.stat.sol. (a) 199 (2003) 9.

[2] E.A. Ekimov, V.A. Sidorov, E.D. Bauer, N.N. Melnik, N.J. Curro, J.D. Thomson and S.M. Stishov, nature 428 (2004) 542.

[3] Y. Takano, M. Nagao, I. Sakaguchi, M. Tachiki, T. Hatano, K. Kobayashi, H. Umezawa and H. Kawarada, Appl. Phys. Lett. 85 (2004) 2851.

[4] E. Bustarret, J. Kačmarčik, C. Marcenat, E. Gheeraert, C. Cytermann, J. Marcus and T. Klein, Phys. Rev. Lett. 93 (2004) 237005.

[5] V.A. Sidorov, E.A. Ekimov, E.D. Bauer, N.N. Mel'nik, N.J. Curro, V. Fritsch, J.D. Thompson, S.M. Stishov, A.E. Alexenko and B. Spitsyn, Diam. Rel. Mater. 14 (2005) 335.

[6] N. Dubrovinskaia, G. Eska, G.A. Sheshin, H. Braun, J. Appl. Phys. 99 (2006) 033903.

[7] V.V. Brazhkin, E.A. Ekimov, A.G. Lyapin, S.V. Popova, A.V. Rakhmanina, S.M. Stishov, V.M. Lebedev, Y. Katayama and K.Kato, Phys. Rev. B 74 (2006) 140502 (R).

[8] L. Vegard, Z. Phys. 5 (1921) 17.

[9] F. Brunet, P. Germi, M. Pernet, A. Deneuville, E. Gheeraert, F. Laugier, M. Burdin and G. Rolland, J. Appl. Phys. 83 (1998) 181.

[10] X. Blase, Ch. Adessi and D. Connétable, Phys. Rev. Lett. 93, 237004 (2004).

[11] L. Boeri, J. Kortus and O.K. Andersen, Phys. Rev. Lett. 93, 237002 (2004).

[12] K.-W. Lee and W.E. Pickett, Phys. Rev. Lett. 93, 237003 (2004).

[13] E. Bourgeois, E. Bustarret, P. Achatz, F. Omnès and X. Blase, Phys. Rev. B 74, 094509 (2006).

[14] N. Troullier, J.L. Martins, Phys. Rev. B 43 (1991) 1993.

[15] S.W. Han, J. Ihm, S.G. Louie et al, Phys. Rev. Lett. 80 (1998) 995.

[16] D. Roundy, M.L. Cohen, Phys. Rev. B 64 (2001) 212103.

[17] D.Connétable, V.Timoshevskii, E.Artacho, X. Blase, Phys. Rev. Lett. 87(2001) 206405.

[18] I. Yokota, J. Phys. Soc. Jpn. 19 (1964) 1487.

[19] T. Klein, P. Achatz, J. Kacmarcik, C. Marcenat, F. Gustafsson, J. Marcus, E. Bustarret, J. Pernot, F. Omnès, Bo E. Sernelius, C. Persson, , A. Ferreira da Silva and C. Cytermann, Phys. Rev. B 75 (2007) 165313. 
[20] M. Mermoux, B. Marcus, F. Jomard, C. Tavares, F. Omnès and E. Bustarret, Diam. \& Relat. Mat. 15 (2006) 572.

[21] M. Cardona and N.E. Christensen Phys. Rev. B 35 (1987) 6182.

[22] H. Umezawa, T. Takenouchi, Y. Takano, K. Kobayashi, M. Nagao, I. Sakaguchi, A. Ishii, M. Tachiki, T. Hatano, Guofang Zhong, M. Tachiki and H. Kawarada., condmat/0503303.

[23] E. Bustarret, P. Achatz, B. Sacépé, C. Chapelier, C. Marcenat, L. Ortéga and T. Klein, Phil. Trans. A366,267 (2008). 
Figure captions

Figure 1

Lattice parameter expansion as a function of the boron atomic density in C:B alloys. Full squares refer to XRD results published in ref. 7 about HPHT crystals, and open squares to relaxed values deduced in ref. 1 from XRD analysis of (100)-oriented epilayers grown by MPCVD. Other open symbols correspond to the results of ab initio calculations on supercells where either isolated boron atoms (circles) or boron pairs (stars) substituted carbon atoms. The straight lines are linear interpolations corresponding to randomly substitutional (dotted) and non substitutional or clustered (dash-dotted) incorporation of boron in diamond.

\section{Figure 2}

Iso-intensity contours around the $\{400\}$ diffraction peak of a $4.3 \mu \mathrm{m}$-thick (100)-oriented heavily B-doped diamond epilayer deposited with an atomic B/C concentration ratio of 1500 ppm. The abscissa axis corresponds to the 2 theta minus omega $(2 \theta-\omega)$ scanning direction while the vertical axis corresponds to the omega $(\omega)$ offset angle (rocking curve).

\section{Figure 3}

Iso-intensity contours around the $\{111\}$ diffraction peak of a miscut (111)-oriented $0.37 \mu \mathrm{m}$ thick heavily B-doped diamond epilayer deposited with a gas flow $\mathrm{CH}_{4} / \mathrm{H}_{2}$ ratio of $0.15 \%$ and an atomic $\mathrm{B} / \mathrm{C}$ concentration ratio of $6000 \mathrm{ppm}$. The abscissa axis corresponds to the 2 theta minus omega $(2 \theta-\omega)$ scanning direction while the vertical axis corresponds to the omega $(\omega)$ offset angle (rocking curve).

Figure 4

Relaxed lattice parameter expansion, deduced from symmetrical XRD measurements of the $\{004\}$ diffraction peaks of both the substrate and the film, as a function of the boron atomic density in two sets of (100)-oriented C:B epilayers grown by MPCVD. The short dashed line corresponds to a linear interpolation of covalent radii whereas long dashes result from linearly interpolating atomic volumes (see text).

\section{Figure 5}

Relaxed lattice parameter expansion, deduced from symmetrical XRD measurements of the $\{111\}$ diffraction peaks of both the substrate and the film, as a function of the boron atomic density in two sets of (111)-oriented C:B epilayers grown by MPCVD. The short dashed line corresponds to a linear interpolation of covalent radii whereas long dashes result from linearly interpolating atomic volumes (see text). 